\title{
Editorial
}

\section{Foreword by the Guest Editors of the RARE special issue}

\section{Corina Constantinescu, Enkelejd Hashorva and Marie Kratz*}

The EU-funded RARE (Risk Analysis, Ruin theory and Extremes) project, co-ordinated by Dr Corina Constantinescu (IFAM, University of Liverpool), addressed rare events and extremes by analysing environmental and financial risks through the use of probabilistic tools and approaches under the scientific co-ordination of Professor Enkelejd Hashorva (HEC, Lausanne) and Professor Marie Kratz (ESSEC, Paris). To achieve this, it established a global network. In total, 20 doctoral and 20 postdoctoral fellows were hired who specialised in (applied) probability, mathematical statistics, stochastic analysis, extremes and financial and insurance mathematics. Partner institutions were 12 leading research centres from Europe, Australia, China, India, Japan, Russia, the United Kingdom and the United States. The network now exceeds 100 researchers from around the world.

RARE focussed on developing a theoretical perspective on the analysis of risk probabilities in case of disasters or extreme shocks for financial or environmental risk pools. It also centred on creating new risk measures and assessing aggregate risk effects. Over 200 scientific articles were submitted for publication, raising overall awareness of rare and extreme events and significantly boosting the visibility of researchers. These papers highlight results such as new concepts in rare events analysis, new risk measures, extreme behaviour analysis, and improvements of existing risk analysis tools and statistical applications.

Knowledge and expertise were continually fostered through university seminars, conferences, workshops and dedicated sessions at international events. A final workshop at La Baule (France) gathered over 60 international experts representing academia and industry from five continents. They discussed a broad variety of future topics, ranging from transformation of uncertainty into measurable risks to the role of regulators, communication and new democratic systems.

To conclude nicely our FP7-RARE project, we have a special RARE issue in Annals of Actuarial Science (AAS), for which researchers working on one of the three topics of RARE (and not necessarily members of the RARE project) were invited to submit a paper. A total of 13 papers have been selected for this issue. The spectrum of papers presented is quite broad, addressing both practical and organisational issues, as well as theoretical advances in risk theory. The main focus is on insurance, which is in line with the goals of an actuarial journal.

An introductory paper, by M. Dacorogna, pictures the new paradigm of insurance management due to the advent of risk-based regulation. He reviews the main changes linked to the necessity of quantifying risk and understanding the return based on it. After this introduction, we grouped the papers that deal with various aspects of regulatory capital. J. Lévy Véhel concentrates on the choice of risk measures imposed by regulators and its consequences in terms of systemic risk. Dynamic risk measures are studied by Y. Shimizu and S.Tanaka in the context of asset performance and regulatory

*Correspondence to: journals@actuaries.org.uk 
capital. Then we move to the context of ruin theory, with a paper by E. Cheung et al. on ruin probabilities with a generalised dependency structure, followed by a paper by R. Xu et al. on dynamic management of capital based on claims frequency. H. Jiang treats the problem of optimality of dividend payments bringing in Lévy processes. We complete this section with a paper by C. Cai et al. proposing an estimator of the survival probability for a Lévy risk model observed at low frequency. The next group of papers treats the important question of dependence in risk models. The first one, by F. Dufresne et al., introduces, in the context of life insurance, a copula approach to model the dependence between lifetimes within a married couple. Vine copulas are used in the next paper by R. Timmer et al. to model the overall balance sheet of a life insurance company to guide asset allocation decisions. M. Tamraz introduces mixture copulas to model the dependence between frequency and severity of insurance claims. In a more theoretical paper, L. Rojas-Nandayapa and W. Xie question the type of asymptotic tail behaviour, light versus heavy, for phase-type scale mixture distributions. Dacorogna et al. derive analytical formulae for risk measures to investigate the convergence of Monte Carlo (MC) simulations for aggregated risk models under standard dependence structure. In the last paper, S. Asmussen looks at conditional MC techniques to improve density estimation and calculations of standard risk measures under various copula structures. 